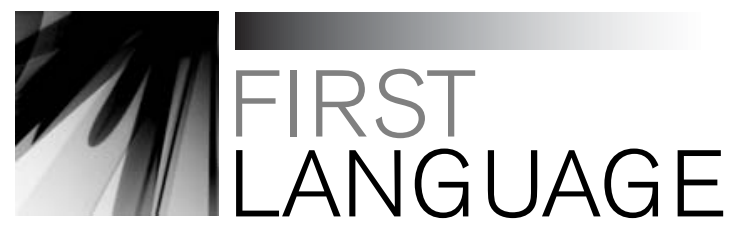

\title{
Lexical and referential influences on on-line spoken language comprehension: A comparison of adults and primary-school- age children
}

\author{
Evan Kidd, The University of Manchester \\ Edith L. Bavin, La Trobe University
}

\begin{abstract}
This paper reports on two studies investigating children's and adults' processing of sentences containing ambiguity of prepositional phrase (PP) attachment. Study 1 used corpus data to investigate whether cues argued to be used by adults to resolve PP-attachment ambiguities are available in child-directed speech. Study 2 was an on-line reaction time study investigating the role of lexical and referential biases in syntactic ambiguity resolution by children and adults. Forty children (mean age $=8 ; 4$ ) and 37 adults listened to V-NP-PP sentences containing temporary ambiguity of PP-attachment. The sentences were manipulated for (i) verb semantics, (ii) the definiteness of the object NP, and (iii) PP-attachment site. The children and adults did not differ qualitatively from each other in their resolution of the ambiguity. A verb semantics by attachment interaction suggested that different attachment analyses were pursued depending on the semantics of the verb. There was no influence of the definiteness of the object NP in either children's or adults' parsing preferences. The findings from the on-line task matched up well with the corpus data, thus identifying a role for the input in the development of parsing strategies.
\end{abstract}

\section{KEYWORDS}

ambiguity resolution; definiteness; lexical semantics; PP-attachment; processing 


\section{VOLUME 27 ISSUE 1}

\section{INTRODUCTION}

Research investigating the time-course of children's on-line sentence processing is important since, logically, in order to learn language children must apply a meaningful analysis to their input (Fodor, 1998). Some researchers propose that children possess the same cognitive mechanisms and resources as adults for processing language (e.g., Meroni \& Crain, 2003), but studies that have directly investigated children's processing indicate that children must, at the very least, fine tune their parsing mechanisms with experience (e.g., Snedeker \& Trueswell, 2004; Traxler, 2002; Trueswell, Sekerina, Hill \& Logrip, 1999). Past studies investigating children's parsing systems have tested structural (Felser, Marinis \& Clahsen, 2003) or lexical effects (Snedeker \& Trueswell, 2004; Traxler, 2002) on comprehension. The current study investigated the contribution of lexical (verb semantics) and referential information (NP definiteness) to children's on-line parsing of sentences containing temporary ambiguity of prepositional phrase attachment (PP-attachment). Consider sentences (1) and (2):

(1) a. The robber blew open a/the safe with a/the new lock.

(1) b. The robber blew open a/the safe with dynamite.

(2) a. The scientist looked at an/the insect with a/the broken wing.

(2) b. The scientist looked at an/the insect with a/the microscope.

The PPs in (1a) and (2a) headed by with are NP-attached; they modify the object NP of the sentence. The PPs in (1b) and (2b) are VP-attached; they modify the verb. Sentences ( $1 a)$ and ( $1 b)$ contain an action verb, whereas ( $2 a)$ and (2b) contain a verb of perception. Studies testing both adults and children have shown that both groups pursue different attachment analyses depending on the semantics of the verb (Kidd \& Bavin, 2005; Spivey-Knowlton \& Sedivy, 1995). The definiteness of the object NP can also potentially affect interpretation. An indefinite determiner (e.g., 'a safe with a new lock') is used to introduce a new referent and so has no bias as to whether a PP might modify the verb or object NP. On the other hand, definite determiners (e.g., 'the safe with the new lock') are generally anaphoric devices that signify reference to a unique entity. Depending on certain discourse conditions they are likely to bias either NP- or VP-attachment differentially, as outlined below.

\section{Theoretical background}

Different theories of sentence processing make different predictions about how sentences (1) and (2) will be processed. We contrast three theories here: the Garden-Path model (Frazier, 1987), Referential Theory (Crain \& Steedman, 1985) and the Constraint-Based approach (MacDonald, Pearlmutter \& Seidenberg, 1994; Trueswell \& Tanenhaus, 1994).

The Garden-Path model postulates a two-stage parsing process whereby a purely syntactic analysis is initially carried out, followed by a second stage which checks this syntactic analysis against semantic/thematic information. The model predicts that the PP will initially be VP-attached due to the implementation of the processing heuristic Minimal Attachment (MA), which predicts that at points of ambiguity the parser chooses the least complex syntactic analysis. PPs that are ultimately 
NP-attached (as in 1a and 2a) must therefore be reanalysed and will result in greater processing cost at the PP. The model does not predict that referential information available on determiners affects initial attachment preference.

Referential Theory postulates an interactive parsing process whereby multiple syntactic analyses are generated in parallel, from which discourse-referential information is used to choose the correct parse. According to Referential Theory, a definite object NP biases VP-attachment when a V-NP-with-NP sequence is presented without a discourse context; that is, in isolation. This follows from the principle of parsimony (Crain \& Steedman, 1985), which postulates that constructing one entity is easier than constructing a set of entities from which to identify a unique token. For instance, for sentence (1), one safe is easier to postulate than a set of safes over which the definite article restricts reference, therefore resulting in a preference for VP-attachment. According to Referential Theory, there is no referential complexity associated with parsing indefinite articles, predicting no additional processing cost associated with an NP-attachment analysis. If a sentence is presented in a supporting context, the principle of referential support (Altmann \& Steedman, 1988) predicts that an NPattachment analysis is no more difficult than a VP-attachment analysis, and that the parser will pursue the interpretation that is best supported by the context.

The Constraint-Based approach (MacDonald et al., 1994; Trueswell \& Tanenhaus, 1994) predicts that the parser will pursue the analysis that is best attested in the input. In particular, it is argued that the parser keeps a record of prior linguistic experience and uses this information to predict the upcoming content of a sentence (Lapata, Keller \& Schulte im Walde, 2001). We next review a selection of empirical studies that bear on the focus of the current research.

\section{Review of the empirical literature}

Analyses of patterns of language use by children and adults show correlations between semantic classes of verbs and the semantic roles expressed by the PPs with which they occur. For instance, in V-NP-with-NP sequences, action verbs most often take a PP that expresses an instrumental or accompaniment role (and are therefore VP-attached), whereas psychological predicates and verbs of perception generally preclude an instrumental interpretation of the PP; they are more likely to occur with a PP that takes an attribute role, as a NP-modifier (Kidd \& Cameron-Faulkner, 2007; Snedeker \& Trueswell, 2004; Spivey-Knowlton \& Sedivy, 1995). Therefore, in natural language there are broad semantic classes of verbs that bias different patterns of PP-attachment.

There is experimental evidence to suggest that such usage patterns guide parsing decisions. Using a self-paced reading task, Spivey-Knowlton \& Sedivy (1995) showed that when adults were presented with V-NP-with-NP sequences like those in (1) and (2), their PP-attachment preference depended on the verb. With sentences containing an action verb, such as blew open, adults showed a preference for VPattachment, that is, the PP was assumed to modify the verb. Therefore sentences like (1b) were read faster than sentences like (1a), where the PP is NP-attached. For sentences containing a psychological predicate or verb of perception, such as look at, participants showed a preference for NP-attachment, although this depended 
on the definiteness of the object NP. When sentences containing psychological predicates and verbs of perception contained an indefinite object NP, there was a preference for NP-attachment, a finding that was also predictable from corpus data. The findings from Spivey-Knowlton \& Sedivy (1995) therefore suggest that in on-line tasks adults are influenced by frequency of distribution. Spivey-Knowlton and Sedivy's data support a constraint-based approach to parsing: participants pursued different syntactic analyses depending on the semantics of the verb, and in a restricted set of cases were affected by the definiteness of the object NP. Based on their findings, Spivey-Knowlton \& Sedivy (1995) identified verb semantics as a strong constraint on PP-attachment and the referential constraint of definiteness of the object NP as a weak constraint.

Trueswell and colleagues have reported several studies investigating children's online processing of PP-attachment ambiguity. Trueswell et al. (1999) presented 5-year-old children and adults with sentences containing temporary ambiguity of PP-attachment, as in (3).

(3) Put the frog on the napkin in the box.

Participants were required to act out the sentence using toy props, and their eyemovements to the toys were recorded as they heard the sentence. There were two experimental conditions: one where there were two tokens of the head referent of the direct object NP (2-referent condition), the frog; and another where there was only one token (1-referent). For instance, in the 2-referent condition there were two frogs, one on a napkin and one not on a napkin, an empty napkin and a box. In the 1-referent condition the frog that was not on the napkin was replaced by a different toy (e.g., a horse). Therefore the 2-referent condition provided participants with the appropriate referential conditions to process the first PP on the napkin as a noun modifier, and therefore the NP the frog as a unique referent. The results showed that although the adults incorporated this referential information into their on-line parse of the sentence, the children did not. Instead, the children were gardenpathed in both conditions; their pattern of eye-movements suggested that they processed the PP on the napkin as the location argument of put, a VP-attachment interpretation. On the basis of these results, Trueswell et al. argued that 5-year-old children base their parsing decisions largely on the lexical information available on the verb; they do not use referential information that is encoded in the language (the definite description the frog) or that is available in the environment (2-referent versus 1-referent). However, the children's results are also consistent with the Garden-Path model, which argues for VP-attachment.

In another eye-tracking study, Snedeker \& Trueswell (2004) presented 5-year-old children and adults with fully ambiguous sentences in three verb bias conditions, one where the verb biased an instrumental interpretation of with (and thus VPattachment), one where the verb biased a modifier interpretation of with (and thus NP-attachment), and one where there was no clear bias for either attachment site (see 4-6).

(4) Tickle the pig with the fan.

(5) Choose the cow with the stick.

(6) Feel the frog with the feather.

(instrument bias)

(modifier bias)

(no bias) 
A verb's bias was determined by the results of a sentence completion study conducted with adults. Once again, the participants were required to act out sentences using toys in either a 1- or 2-referent condition, and their eye-movements to the toys were recorded. The results showed that both children and adults pursued initial interpretations based on the bias of the verb. Crucially, the children did not readily use the referential information available to them in the environment to resolve the ambiguity; that is, there were no reliable differences between the 1-referent and 2-referent conditions. In contrast, the adults did use this referential information. Snedeker and Trueswell did find some evidence in the children's eye-movements to suggest that they were attending to the referential information when the verb had no clear attachment bias (6 above). However, this effect was only present in a subset of the data. Overall, the fact that children pursued different attachment interpretations based on the bias of the verb supports a Constraint-Based account.

Kidd \& Bavin (2005) investigated 3- to 9-year-old children's ability to use lexical (verb semantics) and referential information (object NP definiteness) as cues to resolve PP-attachment ambiguity. In a picture selection study they presented children with both interpretations of fully ambiguous V-NP-with-NP sentences and asked them to indicate their preferred interpretations. Following Snedeker \& Trueswell (2004), there was a verb semantics by attachment interaction, such that sentences containing action verbs were most often VP-attached, and sentences containing verbs of perception were most often NP-attached. The results also showed that the children were sensitive to the constraint of the definiteness of the object NP at age 7 years, but that only 9-year-old children were capable of processing the definite article in a discourse-appropriate manner. That is, only the 9-year-old children understood that the definite article selects a unique referent from a set when presented with a supporting context, thus making more NP-attachment interpretations when the object NP was definite. The 7-year-old children were affected by the definiteness manipulation in the opposite direction: they made more VP-attachment interpretations when the test sentences contained a definite article than when they contained an indefinite article. A potential explanation for this effect is that the 7-year-old children were parsing the sentences purely on the basis of the distributional information available to them in the input, as the pattern of responding follows the corpus data reported by Spivey-Knowlton \& Sedivy (1995). According to this explanation, the 9-year-old children would be able to override this distributional preference by integrating the referential information available to them in the scene with their knowledge of the discourse function of the definite article. This explanation is supported by developmental research investigating the acquisition of the functional properties of the determiner system (e.g, Anderson, Clark \& Mullin, 1994; Karmiloff-Smith, 1979, 1985; Maratsos, 1976), which has shown that children do not use the definite and indefinite article in a discourse appropriate manner until late in development.

Since the studies reported in Kidd \& Bavin (2005) were conducted off-line, the question of whether children are sensitive to the definiteness of the object NP in an on-line processing task is yet to be determined. The current research aimed to extend the findings of Kidd \& Bavin (2005), Snedeker \& Trueswell (2004) and SpiveyKnowlton \& Sedivy (1995) using corpora analyses and a self-paced listening task. 
The objective was to investigate whether children (7-9 years) as well as adults are sensitive to verb semantics and the definiteness of the object NP in V-NP-with-NP sequences, and whether these cues are reliably available in caregiver input to children. In Study 1 we present a corpus study of child-directed speech that investigated the availability of the cues of verb semantics and object NP definiteness to the resolution of PP-attachment ambiguity. In Study 2 we present the results of an on-line self-paced listening task that investigated whether children and adults use these same cues to resolve sentences containing ambiguity of PP-attachment.

\section{STUDY 1}

Study 1 analysed naturalistic speech to children in order to ascertain whether the cues of verb semantics and definiteness are available in child-directed speech. Data from seven corpora were analysed: Adam, Eve and Sarah from the Brown corpus (Brown, 1973); Anne, Mary and Nancy from the La Trobe University corpus (Kidd, 2003); and Brian from the Max Planck Institute for Evolutionary Anthropology dense database corpus (Lieven, Behrens, Spears \& Tomasello, 2003). The details for each child are provided in Table 1.

All V-NP-with-NP sequences where the object NP was headed by either a or the (i.e., pronouns and proper nouns were excluded, along with quantifiers such as some and many, and the demonstrative that) were extracted from each input data set. Despite these restrictions, the constructional pattern is quite common in childdirected speech. Based on the Brian corpus - the richest dataset available to us - we estimate that children hear this construction once every hour of infant-caregiver interaction. Therefore it can be argued that we are dealing with a structural ambiguity that children hear regularly. These target sentences were then coded for (i) verb semantics (action verbs, psychological predicates and verbs of perception, and light verbs), and (ii) definiteness of the object NP. This resulted in 276 tokens. Table 2 presents the frequency of NP- and VP-attached PPs by verb semantic class and definiteness of the object NP for the target sentences.

Overall, action verbs most often occurred with VP-attached PPs (81\%), psychological predicates and verbs of perception most often occurred with NP-attached PPs (89\%), and light verbs showed a general preference for NP-attachment (39\%

Table 1 Details for each child in Study 1

\begin{tabular}{lcc}
\hline Child & \multicolumn{1}{c}{ Source } & Data sampling period \\
\hline Adam & Brown Corpus (USA) & $2 ; 3-4 ; 10$ \\
Eve & Brown Corpus (USA) & $1 ; 6-2 ; 3$ \\
Sarah & Brown Corpus (USA) & $2 ; 3-5 ; 1$ \\
Anne & La Trobe (Australia) & $1 ; 10-3 ; 11$ \\
Mary & La Trobe (Australia) & $1 ; 10-3 ; 11$ \\
Nancy & La Trobe (Australia) & $1 ; 6-3 ; 5$ \\
Brian & MPI (Manchester, UK) & $2 ; 0-2 ; 6$ \\
\hline
\end{tabular}


Table 2 PP-attachment frequencies by verb class and object NP definiteness

\begin{tabular}{lccr}
\hline & NP-attached & VP-attached & Total \\
\hline Action verbs & & & \\
$\quad$ Definite & 9 & 63 & 72 \\
Indefinite & 12 & 27 & 39 \\
Total & 21 & 90 & 111 \\
Psychological and & & & \\
perception verbs & & & \\
Definite & 7 & 1 & 10 \\
Indefinite & 10 & 1 & 9 \\
Total & 17 & 2 & \\
Light verbs & & & 68 \\
$\quad$ Definite & 36 & 32 & 86 \\
Indefinite & 53 & 25 & 146 \\
$\quad$ Total & 89 & 57 & \\
\hline
\end{tabular}

VP-attached, 61\% NP-attached). Tests of proportion were conducted to compare the differences between the frequency of NP-attachment versus VP-attachment for each verb type, controlling for definiteness. The dependent variable was the number of NP-attachment PPs divided by the total number of tokens for each condition, i.e., no. NP-attachments/no. (NP-attachments + VP-attachments). This was computed for each child to control for differences in dialect. The proportions were then tested against a baseline proportion of 0.5 . That is, the null hypothesis was that there was no attachment preference.

For sentences containing action verbs there was a significant preference for VP-attachment when sentences contained either a definite or indefinite object NP (definite: sample proportion $=0.125, z=-6.364, p<0.001, \mathrm{Cl}_{95}[0.049,0.201]$; indefinite: sample proportion $=0.308, z=-2.402, p=0.016, C l_{95}[0.163$, $0.453])$. This suggests that the constraint of verb semantics is present in children's input for this verb class. Whether or not the constraint of object NP definiteness is available is unclear. It is certainly the case that there are proportionally more NPattachments when the sentences contained an indefinite object NP. However, both analyses show a significant preference for VP-attachment, and so any effect of definiteness appears to be minimal.

For sentences containing psychological predicates and verbs of perception there was a significant preference for NP-attachment when sentences contained either a definite or indefinite object NP (definite: sample proportion $=0.875, z=2.399$, $p=0.034, \mathrm{Cl}_{95}[0.646,1.00]$; indefinite: sample proportion $=0.909, z=2.714$, $\left.p=0.007, \mathrm{Cl}_{95}[0.739,1.00]\right)$. This suggests that the constraint of verb semantics is present in the children's input for this verb class. However, since the results for the definite and indefinite conditions were very similar, the constraint of object NP definiteness does not appear to be operative. 
Finally, we conducted the same analyses for sentences containing light verbs. Light verbs such as go, make and copula be make an interesting comparison to the other verb classes because they should have no overwhelming bias for either NP- or VP-attachment. This is because they are largely bleached of semantic content (see Clark, 1996; Goldberg, 1999; Ninio, 1999). If we were to find a strong effect of definiteness it should be for this verb class, as the cue cannot be overwhelmed by the stronger cue of verb semantics. There was no significant bias for either NP- or VP-attachment when the object NP was definite (sample proportion $=0.529$, $\left.z=0.485, p=0.628, \mathrm{Cl}_{95}[0.411,0.648]\right)$. However, there were significantly more NP-attachments when the object NP was indefinite (sample proportion $=0.679$, $\left.z=3.170, p=0.002, \mathrm{Cl}_{95}[0.576,0.783]\right)$. Therefore there is a clear difference in attachment preference that is attributable to the constraint of object NP definiteness when V-NP-with-NP sentences contain light verbs.

\section{Discussion}

The results from the corpus analysis showed that verb semantics reliably predicts patterns of PP-attachment. This supports results presented by Spivey-Knowlton \& Sedivy (1995), who observed the same effect in written corpora. Kidd (2003) and Snedeker \& Trueswell (2004) also reported the same effect in analyses of spoken corpora. The definiteness of the object NP only influenced attachment when the sentences contained light verbs. This is inconsistent with results reported by Spivey-Knowlton and Sedivy, who showed definiteness to affect attachment for sentences containing action verbs and for those that contained psychological verbs. It is likely that this difference is due to the nature of corpora. The present study used spoken language data whereas Spivey-Knowlton and Sedivy used written corpora (Brown corpus; Kucera \& Francis, 1967). The mediums clearly have different referential requirements. In written text the definite article is generally anaphoric; the referent must have been introduced in the text. ${ }^{1}$ In contrast, for spoken language definite reference can be supported through real world context. Thus spoken language interaction provides a situation where it is permissible to introduce a referent using a definite article that refers deictically. When a referent is introduced, a speaker is more likely to provide additional identifying information, such as a PP modifier. This potentially explains why more NP-attachments were observed when sentences contained a definite object NP in our spoken corpora than has been reported in written corpora. We consider this issue further in the General Discussion below.

The conclusion from Study 1 is that verb semantics is available as a cue to the interpretation of sentences containing ambiguity of PP-attachment in the input to children, but that the cue of object NP definiteness is only available in a restricted set of instances. That is, when a V-NP-with-NP sequence contains a light verb, which is an instance when the semantics of the verb is not an informative cue to the construal of with. Showing that a cue is available does not automatically entail that it is used by the parser. In Study 2 we test whether children and adults utilize this information on-line during spoken language comprehension. 


\section{STUDY 2}

The results from Study 1 provide a basis from which to formulate competing hypotheses regarding children's on-line parsing preferences for sentences containing ambiguity of PP-attachment. We conducted an on-line listening study where we presented children (7-9 years) and adults with temporarily ambiguous V-NP-with-NP sentences manipulated for: (i) verb semantics (action verbs versus psychological predicates and verbs of perception); (ii) the definiteness of the object NP (definite versus indefinite); and (iii) PP-attachment site (NP- versus VP-attachment). The on-line nature of the task meant that we were attempting to investigate participants' use of verb semantics and object NP definiteness to determine attachment preference.

The three models of parsing detailed in the Introduction make different predictions regarding the decisions made by the parser at the point of temporary ambiguity the PP. The Garden-Path model (Frazier, 1987) predicts an initial preference for VPattachment regardless of the manipulation of verb semantics and definiteness. This is because the parser is argued not to make use of semantic/pragmatic information in the initial stage of parsing. Statistically speaking, the Garden-Path model predicts a main effect of attachment, with longer listening times predicted for NP-attached PPs over those that are VP-attached (longer listening times being indicative of re-analysis effects). In the absence of a discourse context, Referential Theory predicts a preference for VP-attachment for sentences containing a definite object NP (via the principle of parsimony); there is argued to be no preference when a sentence contains an indefinite object NP. This is predicted for all sentences regardless of verb type. Referential Theory therefore predicts an attachment by definiteness interaction, where participants will experience longer listening times to sentences containing definite object NPs with NP-attached PPs, but no attachment preference for sentences containing indefinite object NPs. The Constraint-Based approach predicts that the parser will pursue the analysis best attested in the input. That is, following the results of Study 1, verb type will act as a strong cue to interpretation but definiteness of the object NP should not strongly influence attachment decisions when sentences contain action verbs and psychological predicates and verbs of perception (we did not test light verbs in Study 2). The Constraint-Based approach therefore predicts a verb by attachment interaction, where action verbs bias VP-attachment and psychological predicates and verbs of perception bias NP-attachment. That is, sentences with action verbs that have VP-attached PPs will be listened to more quickly than those with NPattached PPs. The opposite effect is predicted for sentences containing psychological predicates and verbs of perception. These competing predictions were tested in Study 2 using the auditory moving window technique (Ferreira, Henderson, Anes, Weeks \& McFarlane, 1996).

\section{Method}

\section{Participants}

A total of 77 particiapants completed the task: 40 children aged 7-9 years (mean age $8 ; 4$, range: $7 ; 2-9 ; 11)$ and 37 adults. All children were recruited from three primary 
schools in the Melbourne metropolitan area, Australia. The adult participants were undergraduate and postgraduate students from La Trobe University. All participants were native speakers of Australian English. All the children completed the Recalling Sentences subtest of the CELF-3 (Clinical Evaluation of Language Fundamentals-3; Semel, Wiig \& Secord, 1995), which has been shown to be a reliable screen for language impairment (Conti-Ramsden, Botting \& Faragher, 2001). All scored within the normal range on this task, and so all were included in the final analysis.

\section{Materials}

Twenty-four sentence quadruplets were created, resulting in a total of 96 test sentences (for full list, see Appendix). Each sentence had a VP-attachment and an NP-attachment version, with each attachment version having a definite NP in an object role as well as an indefinite NP version. All the verbs were familiar to children. Although age of acquisition ratings were not available for all verbs, a check on the MRC database (Wilson, 1988) showed that all verbs appeared regularly. ${ }^{2}$ For eight of the verbs the object role was oblique (as in 'look at NP'). For the other verbs it was the direct object. One sentence quadruplet with the four possible versions is shown in sentences (7a) to (7d). All sentence quadruplets were matched for syllable length (see Appendix). In (7) the length of the PP in each of these four sentences was five syllables.

(7a) The girl/looked at/the boy/with the basketball/because she/wanted to play. (NP-attachment, definite object NP)

(7b) The girl/looked at/the boy/ with much excitement/because she/wanted to play. (VP-attachment, definite object NP)

(7c) The girl/looked at/a boy/with a basketball/because she/wanted to play. (NP-attachment, indefinite object NP)

(7d) The girl/looked at/a boy/ with much excitement/because she/wanted to play. (VP-attachment, indefinite object NP)

The slashes in (7a-d) denote the segments ('windows') into which the sentences were divided for presentation. The content of the sentences in each quadruplet differed in the third and fourth segments only.

Spivey-Knowlton \& Sedivy (1995) showed that the determiner within the PP does not affect attachment preferences. Thus, to ensure that the sentences sounded natural, in some sentences the determiner within the PP was varied, as shown in (7a) and (7c). For sentences containing action verbs, 10 of 12 PPs encoded an instrument role for a VP-attachment interpretation (as in Appendix sentence 1b). The other two encoded manner (see Appendix sentence 9) and accompaniment (sentence 10), respectively. For the 12 psychological verbs and verbs of perception, nine of the PPs encoded manner for a VP interpretation (as in sentence 13b), since VP-attached PPs typically take this role for psychological verbs and verbs of perception (Spivey-Knowlton \& Sedivy, 1995). The remaining three VP-attached PPs for psychological verbs and verbs of perception encoded an instrument (as in sentence 22b). Once again, the sentences were constructed in this manner to make them sound more natural. For instance, the VP-attachment version of the sentence containing the action verb read contained a PP encoding accompaniment because this is a more plausible and common event than, for instance, 
reading something with an instrument (e.g., a magnifying glass). We point out that no study has systematically investigated the role of thematic fit between verbs and the different semantic roles with can take in the V-NP-with-PP ambiguity; the theoretical and empirical focus to date has been on syntactic attachment without reference to the fact that a VP-attached with-phrase can encode multiple roles. We leave this issue for further exploration, but point out that previous studies using similar methodologies have constructed their test sentences in a similar manner.

The stimulus items were recorded directly into the digital editing program Sound Forge (Sonic Foundary) at 44,100 Hz in 16-bit Mono. A female native speaker of Australian English recorded the audio stimuli. In order to control for prosodic cues, each segment was recorded in isolation and out of sequence (Ferreira, Anes \& Horine, 1996). This was done because prosodic cues, like discourse context, were not under investigation. As a further control, segments used more than once were recorded once only. The experiment was presented via headphones using E-prime (Psychology Software Tools). Participants controlled the presentation of the sentence segments via a button box.

\section{Procedure}

Adult participants were tested individually in a quiet room. The auditory moving window technique was used (Ferreira, Henderson, et al., 1996). Participants were informed that they would hear some sentences over headphones, but that the sentences were broken up into smaller 'bits'. They were told that to hear each bit of the sentence they had to press a white button on the button box. Participants were also told that at the end of each sentence there would be a post-sentence question for which they had to answer 'yes' or 'no'. A 'yes' answer required participants to press a green button; a 'no' answer required participants to press a red button. The adults were told that the task was designed to test children's language comprehension, and that it was important that they listen to the sentences carefully so that they could answer the post-sentence questions correctly. The questions probed the truth-value of information contained in various parts of the sentence. For instance, the question for (7a), above, was 'Did the girl want to play?' Two questions were constructed for each sentence, although only one question followed any one sentence. The alternative question for (7a) was 'Did the boy have a basketball?' Participants completed four practice items before moving into the test phase of the experiment.

Children were tested individually in a quiet area of their school. Children completed the self-paced listening task in two blocks. Between the two blocks they completed the recalling sentences task. This served to break up the task and aimed to avoid fatigue effects, as well as screen for language problems. After the children had completed the recalling sentences task they were given the option of completing the second block of the self-paced listening task; all children happily accepted. As with the adults, the children were told that they would hear sentences over the headphones, but that the sentences were broken into 'bits'. Each child was encouraged to provide an example sentence for illustration. For instance, one child provided the example, 'Once there was a cat and it was pretty.' The experimenter then discussed how the sentence could be broken up. Using the example sentence provided by the child, it was explained that after pressing the white button on the button box the first 
bit of the sentence would be heard, and that subsequent segments would be heard each time the button was pressed. It was explained that at the end of the sentence there would be a question about what had been heard and an example was given. For the sample sentence 'Once there was a cat and it was pretty', the example question was 'Was the cat pretty?' The buttons for the post-sentence questions were then explained. Children sat facing the experimenter. The total testing time for the children was approximately 40 minutes. However, since the testing time was divided into three blocks (two blocks of the self-paced listening and the recalling sentences task), the children maintained attention throughout the session. Overall, the children appeared to enjoy the task, and had no problem understanding what was expected of them.

Each participant heard only one sentence from each quadruplet, a total of 24 test sentences in total, interspersed between eight fillers. Eight lists of sentences were created: four containing sentences with a definite object NP and four containing sentences with an indefinite object NP. Each child heard sentences from one list. Therefore object NP definiteness was a between-subjects variable; verb semantics was within-subjects. Sentences in a list were presented randomly by E-prime. The delay between button presses was calculated to the nearest millisecond (ms) by the program.

\section{Scoring}

To control for any length differences at the critical PP a difference time score (DT) was computed; the actual duration of each recorded segment was subtracted from the total listening time to that segment, as measured by the button presses. For example, the total duration of the PP in (7a), above, was $1410 \mathrm{~ms}$. If a participant listened to this segment for $1600 \mathrm{~ms}$ (i.e., the time between button presses), the DT was calculated as $1600-1410=190 \mathrm{~ms}$. The use of DT as a dependent variable followed Ferreira, Henderson, et al. (1996).

Since participants listen to the segment and process it before pressing the button for the next segment, DTs should be positive. However, it was possible to have a negative DT if a participant pressed the button before the offset of the completion of a segment. If this cut-off was before the end of the final word in the PP the trial was treated as missing data.

\section{Results}

Both the children and the adults answered a majority of the post-sentence questions correctly (children $=94 \%$, adults $=97 \%$ ). An outlier procedure was employed. For each participant, DTs greater than 2.5 standard deviations from the mean were corrected to that value. DTs greater than 2000 ms were treated as missing data. This accounted for $3.2 \%$ of the data for adults, and $4.6 \%$ of the data for the children. The results are first presented for the critical segment (ambiguous PP) and then for the subsequent segments. Mean DTs (and SEs) for the critical segment for each condition for the adult participants are presented in Fig.1a; the results for the children are presented in Fig. 1b. In these figures, 'ACT' stands for sentences that contained action verbs and 'PSY' stands for sentences that contained psychological verbs and verbs of perception. 'DEF' stands for sentences that contained a definite 
object NP, and 'INDEF' stands for sentences that contained an indefinite object NP. Therefore, 'ACT-DEF' represents sentences that contained an action verb and a definite object NP. Figures $1 \mathrm{a}$ and $1 \mathrm{~b}$ show that, when sentences contained action verbs, adults and children had longer DTs to the NP-attached PPs than to the VP-attached PPs. The magnitude of the difference was greater for the adults than for the children. For sentences containing psychological predicates and verbs of perception (PSY) there was no discernable difference between attachment conditions. Object NP definiteness did not appear to affect attachment in either verb class condition.

Each group's data were analysed separately to avoid any spurious effects that may be observed were the data combined. The analysis of the adult data is presented first, followed by the analysis of the children's data. For all analyses the DTs serve as the dependent variable.

\section{Analysis of adult data}

A 2 (verb) $\times 2$ (definiteness) $\times 2$ (attachment) ANOVA was conducted for both participants $\left(F_{1}\right)$ and items $\left(F_{2}\right)$. There was a significant main effect for attachment

(a)

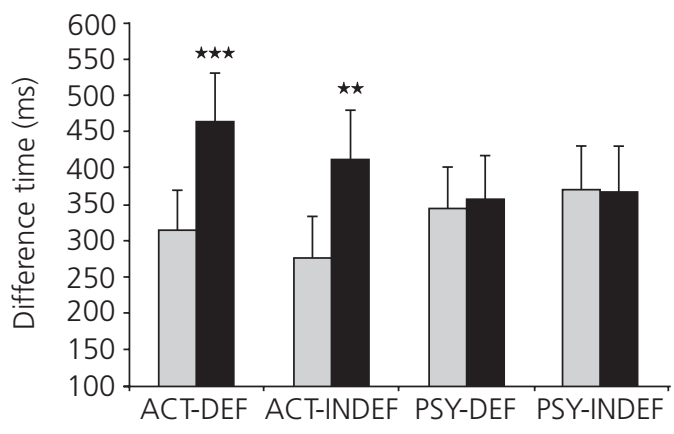

(b)

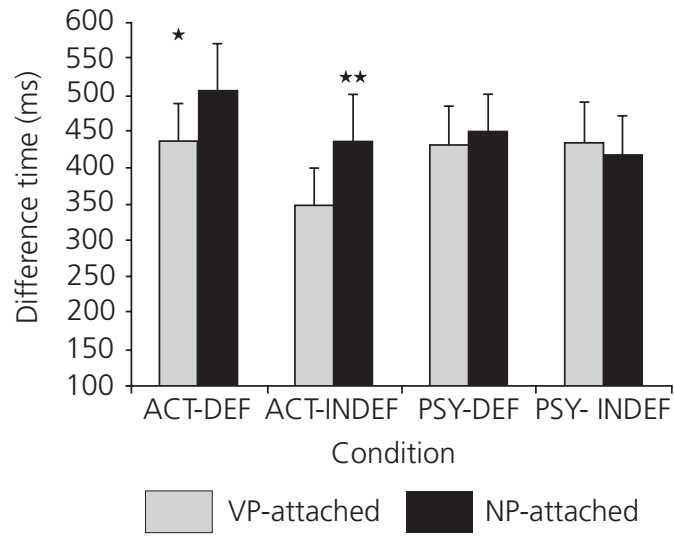

Figure 1 Mean difference times (in ms) and standard errors to the critical segment for the adult (1a) and child (1b) participants (*** $p<0.001,{ }^{* *} p<0.01$, $\left.{ }^{*} p<0.05\right)$; the bar labels are explained in the text 
$\left(F_{1}(1,35)=11.39, p=0.002\right.$, partial $\eta^{2}=0.25 ; F_{2}(1,22)=4.93, p=0.037$, partial $\left.\eta^{2}=0.18\right)$, suggesting that the adults processed sentences containing VP-attached PPs faster than NP-attached PPs overall. However, this effect was subsumed by a significant verb by attachment interaction, suggesting that verb type affected attachment $\left(F_{1}(1,35)=12.37, p=0.001\right.$, partial $\eta^{2}=0.26 ; F_{2}(1,22)=5.79, p=0.025$, partial $\left.\eta^{2}=0.21\right)$. This effect was due to the fact that DTs to NP-attached PPs were significantly longer than to VP-attached PPs in sentences containing action verbs (all effects significant by subjects and items), but DTs to NP- and VP-attached PPs in sentences containing psychological predicates and verbs of perception did not differ significantly. No other effects were significant.

We analysed the segments following the critical segment to determine if there were any spillover effects. There were no reliable significant effects after the critical region.

\section{Analysis of children's data}

The children's data showed a very similar pattern of results. A 2 (verb) x 2 (definiteness) $\times 2$ (attachment) ANOVA was conducted for both participants $\left(F_{1}\right)$ and items $\left(F_{2}\right)$. Once again, a significant main effect for attachment $\left(F_{1}(1,38)=4.98\right.$, $p=0.032$, partial $\eta^{2}=0.12 ; F_{2}(1,22)=2.93, p=0.098$, partial $\eta^{2}=0.12$ ) was subsumed by a verb by attachment interaction in the same direction as the adult data $\left(F_{1}(1,38)=5.61, p=0.023\right.$, partial $\eta^{2}=0.13 ; F_{2}(1,22)=5.32, p=0.031$, partial $\left.\eta^{2}=0.20\right)$. This effect was again due to the fact that DTs to NP-attached PPs were significantly longer than to VP-attached PPs in sentences containing action verbs (all effects significant by subjects and items), but DTs to NP- and VP-attached PPs in sentences containing psychological predicates and verbs of perception did not differ significantly. There were no other significant effects at the critical segment.

An analysis of the segment after the critical region yielded a significant main effect for verb type $\left(F_{1}(1,38)=41.28, p<0.001\right.$, partial $\eta^{2}=0.52 ; F_{2}(1,22)=12.42$, $p=0.002$, partial $\left.\eta^{2}=0.36\right)$. This was due to the fact that the children took longer to process this segment when sentences contained a psychological predicate or verb of perception than when it contained an action verb. No other significant effects were observed.

\section{Comparison of adults and children}

The two groups' data were combined to explore whether there were any qualitative differences in how the children and adults parsed the test sentences. At the critical segment the main effect of age was significant by items but not by participants $\left(F_{1}(1,73)=1.72, p=0.194\right.$, partial $\eta^{2}=0.02, n s ; F_{2}(1,44)=8.17, p=0.006$, partial $\left.\eta^{2}=0.16\right)$. This was due to the fact that adults processed this segment faster than the children did. There were no reliable interactions involving the group variable.

The regions following the critical segment were also analysed using group as an independent variable. No significant interaction was found in the segment immediately following the critical region, although there was a main effect of age $\left(F_{1}(1,73)=16.98, p<0.001\right.$, partial $\eta^{2}=0.19 ; F_{2}(1,44)=39.66, p<0.001$, 
partial $\left.\eta^{2}=0.47\right)$. Adults processed this segment significantly faster than the children. There was also a main effect for verb $\left(F_{1}(1,73)=50.56, p<0.001\right.$ partial $\eta^{2}=0.41 ; F_{2}(1,44)=14.79, p<0.001$, partial $\left.\eta^{2}=0.25\right)$, showing that this post critical segment was processing faster in sentences containing action verbs than those containing psychological predicates and verbs of perception by both children and adults when their data were combined. There were no significant effects at the final segment. ${ }^{3}$

\section{Discussion}

The results of Study 2 suggest that children and adults processed the test sentences in a similar way. The pattern of results are best accounted for by the Constraint-Based approach; the verb by attachment effect observed for children and adults shows that both age groups were influenced by verb semantics in predicting the content of the sentence after they had heard the verb, and their analyses differed according to verb type. This finding is consistent with those reported by Spivey-Knowlton \& Sedivy (1995) and Snedeker \& Trueswell (2004). The results are not consistent with predictions made by either the Garden-Path model or Referential Theory, both of which do not predict the early use of semantic information carried on the verb in the current experimental context.

The main departure from Spivey-Knowlton \& Sedivy (1995) is that no attachment preference was found for sentences containing psychological predicates and verbs of perception. This was a rather modest, although robust, effect in their data. ${ }^{4}$ In the present paper, the results of Study 1 suggest that although this constraint is available in the ambient language, psychological predicates and verbs of perception do not occur very frequently in the target structure. Therefore, although the constraint has high cue reliability, it has low cue availability, which potentially weakens its utility on-line.

It is also possible that the difference in method between the two studies can explain the difference in results. Although in self-paced reading a word disappears after the next word appears, the stimulus is perceptually available to the reader until they decide to progress to the next word, which is not the case in self-paced listening. Therefore the transient nature of auditory stimuli could explain why the participants in the present study did not immediately commit to a particular syntactic analysis when sentences contained psychological verbs and verbs of perception. Note that this may only apply when a cue is relatively weak, since in the present study the attachment effect observed for sentences containing action verbs was very large.

The definiteness of the object NP did not affect either adults' or children's processing of the critical segment. This is in contrast to the reading time data reported by Spivey-Knowlton \& Sedivy (1995), and does not follow the predictions of Referential Theory either. Note, however, that it does broadly follow the predictions made by the Constraint-Based account. The results from Study 1 showed that definiteness does not reliably predict attachment preferences when V-NP-with-NP sentences contain either action verbs or psychological predicates and verbs of 
perception. This suggests that this distinction is not marked in spoken language, and is therefore not used as a cue by the parser in the auditory domain.

\section{GENERAL DISCUSSION}

In this paper we explored the role that lexical and referential factors play in the resolution of sentences containing temporary ambiguity of PP-attachment. The results from our corpus study suggested that although the cue of verb semantics was available to children in the input, the definiteness of the object NP, which has been shown to be a reliable cue in adult written corpora, was not a reliable cue to attachment in the children's input. The results from an on-line self-paced listening task suggested that children and adults parsed the sentences in a similar manner. Both groups' attachment decisions were affected by the semantics of the verb but not by the definiteness of the object NP. This pattern of results is best explained by a Constraint-Based model of parsing.

The use of verb semantics as a cue to ambiguity resolution is well attested in studies of both children's and adults' parsing (e.g., Kidd \& Bavin, 2005; Snedeker \& Trueswell, 2004; Spivey-Knowlton \& Sedivy, 1995). The results of the current research add to this literature. These findings argue against a parsing heuristic that is blind to the semantic content of lexical items, such as MA in the Garden Path model (Frazier, 1987). The results raise the question of how children acquire these lexical biases. One possible way is if children keep track of the kinds of syntactic environments in which a verb occurs; that is, if they store probabilistic distributional information in the lexical entry for the verb. This suggestion is consistent with adult processing studies that have shown the parser to make use of lexical frequency information to postulate syntactic structure at points of temporary ambiguity (e.g., Garnsey, Pearlmutter, Myers \& Lotocky, 1997; Trueswell, 1996; Trueswell, Tanenhaus \& Kello, 1993). With respect to young children, Kidd, Lieven \& Tomasello (2006) have shown that children as young as 3-years use lexical frequency information encoded on the verb to predict upcoming structure. Young children appear to be very adept at performing distributional analyses over their input (e.g., Saffran, Aslin \& Newport, 1996), and so children appear to possess the right kind of mechanism that would enable them to register frequency statistics. Once children perceive similarity in distributions they can begin to form subclasses of verbs. These subclasses will roughly correspond to the semantic groupings argued to affect processing in the current paper.

Therefore we argue that the effect of verb semantics observed here and elsewhere derives from distributional learning. However, it is an open question whether or not these frequency statistics are consulted by the parser on-line. It is entirely possible that verb semantics is an informative enough cue once subclasses of verbs are formed, and that frequency information need not be consulted on-line (see recent work by Hare, McRae \& Elman, 2003, 2004). It is difficult to tease apart these two possibilities, since verb semantics and frequency of distribution are inherently confounded in natural languages (see Fisher, Gleitman \& Gleitman, 1991). That is, semantic classes of verbs tend to behave similarly syntactically. The results of our corpus study demonstrate this 
phenomenon. Future research could attempt to tease apart this issue, potentially by training children on novel verbs, where both the semantic content of the verb and its distributional properties can be controlled directly the experimenter.

The finding that the definiteness of the object NP was not strongly available in the input to children and was not used a cue to resolve the attachment ambiguity in Study 2 suggests that this constraint is not as strong in spoken language as in written language. That the definiteness effect was not observed in either study is troubling for Referential Theory. It suggests that what has been argued to be a domain-independent referential complexity effect may in fact be attributable to discourse-pragmatic factors independent of structure building. Consider the following explanation: a definite article is more likely to be used to reactivate an already mentioned discourse referent, while indefinites introduce new referents. When used as modifiers PPs add information about a referent. If a referent has already been adequately established in the context, as is most often the case when definite articles are used, there is less need to provide additional information when the referent is reactivated. However, when a referent is introduced the need to provide additional information about this referent is heightened. One way to add information about a referent is to use a PP modifier. Thus the different discourse functions of the definite and indefinite articles provide an alternative explanation to the attachment effect found by Spivey-Knowlton \& Sedivy (1995). Why is this effect not observed in spoken language? As argued above, the discourse pressures of spoken language are different to those of written language. In the case of early caregiverchild discourse, reference is largely restricted to the immediate physical environment (Snow, 1977), just the kinds of situations where (i) definite determiners can be used deictically and not anaphorically, or (ii) there is an assumption of mutual knowledge making the communicative need to provide additional modifying information redundant.

The results from Study 2 suggest that the constraint of definiteness might not be strong even for adults in spoken language. Results from studies of referential communication show that neither older children nor adults reliably use definiteness in a discourse appropriate manner. For instance, Brown-Schmidt, Campana \& Tanenhaus (2005) showed that almost half of their adult participants' definite expressions did not identify a unique referent. Additionally, Anderson et al. (1994) and Anderson \& Boyle (1994) showed that children and adults do not reliably introduce new referents using the indefinite article. They showed that both participant groups used other linguistic conventions available to them to establish common ground. Anderson and colleagues suggest that definiteness is not a reliably used discourse convention in spoken language because the definite and indefinite articles lack perceptual saliency and so are often difficult to distinguish during the online computational pressures of spoken discourse. This point is particularly pertinent for children who are still learning the different functions of this system: even if the definite and indefinite articles are reliably used for different functions in spoken discourse directed to children, they must still identify the phonological form of each in the incoming speech stream. Bard \& Anderson (1984), who investigated the intelligibility of parental speech, have shown that this is a non-trivial proposition for children. The suggestion is that multiple factors 
conspire to prevent definiteness from being utilized as a constraint on on-line spoken language comprehension.

The results of the current study can be interpreted as providing support for a Constraint-Based model of parsing (e.g., MacDonald et al., 1994) because verb semantics was found to be the major influencing factor for PP attachment. Proponents of structure-based parsing models often criticize some methods for not being sensitive enough measures of the 'first' parse. It is possible that the self-paced listening task is not a sensitive enough on-line measure to show if participants initially follow a structurally-biased parse and then rapidly revise this interpretation during reanalysis. However, were this to be the case we would expect, at the very least, a qualitatively similar pattern of DTs across verb conditions, which we did not observe.

On the Constraint-Based approach, children are argued to forge parsing preferences by accumulating probabilistic cues to interpretation that vary in strength. Thus a crucial role is identified for children's experience with language as a determinant of their parsing preferences; there is argued to be emergent continuity between syntactic processing and syntactic acquisition. That is, the basic architecture of the human sentence processing mechanism is argued to be available to the child from birth, but differences in children's and adults' exposure to language means that there will be points in development where children will process language differently to adults, since strong constraints that have high cue reliability will be more strongly relied upon than weaker constraints (as in Snedeker \& Trueswell's (2004) 5-year-olds; see also Trueswell et al., 1999). The present research has shown a degree of continuity between the language that children hear and the manner in which they process temporary syntactic ambiguities. One potential criticism of our data is that the corpus data from Study 1 contains language spoken to children much younger than the children who participated in Study 2. We accept this criticism of the data, and acknowledge that the children in Study 2 would have a wider range of experience with language (e.g., through more complex input and through text). However, we point out that our child and adult participants showed a similar pattern of results, which were largely consistent with the results from the corpus analysis. Therefore we argue that the connection we have made between the results of the two studies is warranted.

The auditory moving window technique is a useful method for investigating children's sentence processing. It has the advantage of enabling researchers to localize processing effects, and as such identify differences in processing incrementally, as they occur (i.e., at different 'windows'). Therefore it is more sensitive than on-line listening methods that have been traditionally used in child language research, such as grammatical judgement, where reaction times are recorded at the end of the sentence (e.g., von Beger, Wulfleck, Bates \& Fink, 1996). Another major advantage is that the technique does not require reading, therefore multiple age groups can be compared without confounding reading ability. The children in the present study had no difficulty understanding the requirements of the task. However, Kidd (2003) reported that many 5-year-olds could not complete the task in a manner that yielded significant information about their on-line parsing. As the linguistic stimuli are presented in chunks, it does sound a little unnatural. However, our participants did not seem to have a problem with this feature of the task. 
Using a different methodology from that of Trueswell et al. (1999) and Snedeker \& Trueswell (2004), the current study has shown that children are sensitive to verb semantics in the resolution of syntactic ambiguity and thus the findings show that lexical information affects parsing. No referential effects based on NP definiteness were found in the current study, but nor were they found for the adults. We argued that the failure to observe an effect for the manipulation of definiteness was due to the fact that the current research was concerned with spoken rather than written language. Further research comparing the two domains is needed to investigate these differences.

\section{ACKNOWLEDGEMENTS}

This research was supported in part by an Australian Postgraduate Award to Evan Kidd while at La Trobe University, as well as a Postdoctoral Research Fellowship from the Max Planck Institute for Evolutionary Anthropology. Data collection for the Australian corpus was supported by a large ARC grant to Edith L. Bavin. We would like to thank the following people for their assistance and/or helpful comments: Kerry Bratby, Sheree Hegarty, Jarrad Lum, Leanne Kelly, Jesse Snedeker, Thea Cameron-Faulkner, Franklin Chang, Cindy Fisher and Andrew Stewart.

\section{NOTES}

1. A definite article will refer to an entity not introduced in prior written text in very limited and marked situations, such as when the NP designates a fixed property bound by contextual properties such as time, place and situation (e.g., 'The prime minister' or 'The bathroom'). For further discussion, see Epstein, 2001.

2. Although overall frequency aids lexical access, it is certainly not the case that it is a good predictor of syntactic processing. Kidd et al. (2006) showed that 3- to 5-year-old children's syntactic processing was predicted by frequency of distribution, not by raw frequency of occurrence (see also MacDonald \& Shillcock, 2001).

3. We also analysed our results with the PPs that encoded different roles removed, such that all sentences with action verbs had VP-attached PPs encoding an instrument, and all sentences containing psychological predicates and verbs of perception had VP-attached PPs that encoded manner. The results stayed essentially the same. For children there was a verb by attachment interaction at the critical segment $\left(F_{1}(1,38)=6.38, p=0.016\right.$, partial $\eta^{2}=$ $0.144 ; F_{2}(1,17)=3.72, p=0.071$, partial $\left.\eta^{2}=0.179\right)$. For adults there was also a verb by attachment interaction at the critical segment $\left(F_{1}(1,35)=23.86, p<0.001\right.$, partial $\eta^{2}=$ $0.406 ; F_{2}(1,17)=4.126, p=0.058$, partial $\left.\eta^{2}=0.195\right)$. Removing these items strengthen the $F_{1}$ verb by attachment effect for both children and adults; in particular, the adults show a tendency to pursue an NP-attachment analysis when sentences contained psychological predicates and verbs of perception $(p=0.10)$. However, the items analysis became unstable because removing the additional items resulted in a loss of statistical power.

4. The effect size for their verb by determiner interaction in their study examining psychological verbs was $d=0.77$ (Cohen's $d$; Cohen, 1988), which, although Cohen interprets as being medium to large, still results in half of the distribution of reaction times overlapping with each other. That is, patterns of approximately half of the individual participant's reaction times will not follow the group trend, although the group data show a significant effect. For comparison, the effect size for the same result in our data (adults only) was $d=0.11$. 


\section{REFERENCES}

Altmann, G. \& Steedman, M. (1988). Interaction with context during human sentence processing. Cognition, 30, 191-238.

Anderson, A. H. \& Boyle, E. A. (1994). Forms of introduction in dialogues: Their discourse contexts and communicative consequences. Language and Cognitive Processes, 9, $101-122$.

Anderson, A. H., Clark, A. \& Mullin, J. (1994). Introducing information in dialogues: Forms of introduction chosen by young speakers and the responses elicited from young listeners. Journal of Child Language, 18, 663-687.

Bard, E. G. \& Anderson, A. H. (1984). The unintelligibility of speech to children. Journal of Child Language, 10, 1-18.

Brown, R. (1973). A first language. Cambridge, MA: Harvard University Press.

Brown-Schmidt, S., Campana, E. \& Tanenhaus, M. K. (2005). Real-time reference resolution by naïve participants during a task-based unscripted conversation. In J. C. Trueswell \& M. K. Tanenhaus (Eds), World-situated language processing: Bridging the language as product and language as action traditions (pp. 153-171). Cambridge, MA: MIT Press.

Clark, E. V. (1996). Early verbs, event-types, and inflections. In C. E. Johnson \& J. H. V. Gilbert (Eds), Children's language, Vol. 9 (pp. 61-73). Mahwah, NJ: Erlbaum.

Cohen, J. (1988). Statistical power analysis for the behavioral sciences (2nd ed.). Hillsdale, NJ: Erlbaum.

Conti-Ramsden, G., Botting, N. \& Faragher, B. (2001). Psycholinguistic markers for Specific Language Impairment (SLI). Journal of Child Psychology and Psychiatry and Allied Disciplines, 42, 741-748.

Crain, S. \& Steedman, M. (1985). On being led up the garden path: The use of context by the psychological syntax processor. In L. K. D. Dowty \& A. Zwicky (Eds), Natural language parsing (pp. 320-358). Cambridge: Cambridge University Press.

Epstein, R. (2001). The definite article, accessibility, and the construction of discourse referents. Cognitive Linguistics, 12, 333-378.

Felser, C., Marinis, T. \& Clahsen, H. (2003). Children's processing of ambiguous sentences: A study of relative clause attachment. Language Acquisition, 11, 127-163.

Ferreira, F., Anes, M. D. \& Horine, M. D. (1996). Exploring the use of prosody during language comprehension using the auditory moving window technique. Journal of Psycholinguistic Research, 25, 273-290.

Ferreira, F., Henderson, J. H., Anes, M. D., Weeks, P. A. \& McFarlane, D. K. (1996). Effects of lexical frequency and syntactic complexity in spoken language comprehension: Evidence from the auditory moving window technique. Journal of Experimental Psychology: Learning, Memory, and Cognition, 22, 324-335.

Fisher, C., Gleitman, L. R. \& Gleitman, H. (1991). On the semantic content of subcategorization frames. Cognitive Psychology, 23, 331-392.

Fodor, J. D. (1998). Parsing to learn. Journal of Psycholinguistic Research, 27, 285-319.

Frazier, L. (1987). Sentence processing: A tutorial review. In M. Coltheart (Ed.), Attention and performance XII: The psychology of reading (pp. 559-586). Hillsdale, NJ: Erlbaum.

Garnsey, S. M., Pearlmutter, N. J., Myers, E. \& Lotocky, M. A. (1997). The contributions of verb bias and plausibility to the comprehension of temporarily ambiguous sentences. Journal of Memory and Language, 37, 58-93.

Goldberg, A. (1999). The emergence of the semantics of argument structure constructions. In B. MacWhinney (Ed.), The emergence of language (pp. 197-212). Mahwah, NJ: Erlbaum.

Hare, M., McRae, K. \& Elman, J. (2003). Sense and structure: Meaning as a determinant of verb subcategorization preferences. Journal of Memory and Language, 48, 281-303. 
Hare, M., MacRae, K. \& Elman, J. (2004). Admitting that verb sense into corpus analyses makes sense. Language and Cognitive Processes, 19, 181-224.

Karmiloff-Smith, A. (1979). A functional approach to child language. Cambridge: Cambridge University Press.

Karmiloff-Smith, A. (1985). Language and cognitive processes from a developmental perspective. Language and Cognitive Processes, 1, 61-85.

Kidd, E. (2003). An investigation into children's sentence processing: A developmental perspective. Unpublished PhD thesis, La Trobe University.

Kidd, E. \& Bavin, E. L. (2005). Lexical and referential cues to sentence interpretation: An investigation of children's interpretations of ambiguous sentences. Journal of Child Language, 32, 855-876.

Kidd, E. \& Cameron-Faulkner, T. (2007). The acquisition of the multiple senses of with. Linguistics, 45 (in press).

Kidd, E., Lieven, E. \& Tomasello, M. (2006). Examining the role of lexical frequency in children's acquisition and processing of sentential complements. Cognitive Development, 21, 93-107.

Kucera, H. \& Francis, W. (1967). Computational analysis of present day American English. Providence, Rl: Brown University Press.

Lapata, M., Keller, F. \& Schulte im Walde, S. (2001). Verb frame frequency as a predictor of verb bias. Journal of Psycholinguistic Research, 30, 419-435.

Lieven, E., Behrens, H., Spears, J. \& Tomasello, M. (2003). Early syntactic creativity: A usagebased approach. Journal of Child Language, 30, 333-370.

MacDonald, M., Pearlmutter, N., Seidenberg, M. (1994). Lexical nature of syntactic ambiguity resolution. Psychological Review, 101, 676-703.

MacDonald, S. A. \& Shillcock, R. C. (2001). Rethinking the word frequency effect: The neglected role of distributional information in lexical processing. Language and Speech, 44, 295-323.

Maratsos, M. (1976). The use of definite and indefinite reference in young children. Cambridge: Cambridge University Press.

Meroni, L. \& Crain, S. (2003). On not being led down the kindergarten path. In B. Beachley, A. Brown \& F. Conlin (Eds), Proceedings of the 27th annual Boston University conference on language development (pp. 531-544). Somerville, MA: Cascadilla Press.

Ninio, A. (1999). Pathbreaking verbs in syntactic development and the question of prototypical transitivity. Journal of Child Language, 26, 619-653.

Saffran, J., Aslin, R. \& Newport, E. (1996). Statistical learning by 8-month-old infants. Science, 274, 1926.

Semel, E., Wiig, E. \& Secord, W. (1995). Clinical evaluation of language fundamentals (3rd ed.). San Antonio: Psychological Corporation.

Snedeker, J. \& Trueswell, J. C. (2004). The developing constraints on parsing decisions: The role of lexical-biases and referential scenes in child and adult sentence processing. Cognitive Psychology, 49, 238-299.

Snow, C. (1977). The development of conversation between mothers and babies. Journal of Child Language, 4, 1-22.

Spivey-Knowlton, M. \& Sedivy, J. C. (1995). Resolving attachment ambiguities with multiple constraints. Cognition, 55, 227-267.

Traxler, M. (2002). Plausibility and subcategorization preference in children's processing of temporarily ambiguous sentences: Evidence from self-paced reading. Quarterly Journal of Experimental Psychology, 55A, 75-96.

Trueswell, J. C. (1996). The role of lexical frequency in syntactic ambiguity resolution. Journal of Memory and Language, 35, 566-585. 


\section{VOLUME 27 ISSUE 1}

Trueswell, J. C., Sekerina, I., Hill, N. M. \& Logrip, M. L. (1999). The kindergarten path effect: Studying on-line sentence processing in young children. Cognition, 73, 89-134.

Trueswell, J. C. \& Tanenhaus, M. K. (1994). Toward a lexicalist framework for constraint-based syntactic ambiguity resolution. In C. Clifton Jr., L. Frazier \& K. Rayner (Eds), Perspectives on sentence processing (pp. 155-179). Hillsdale, NJ: Erlbaum.

Trueswell, J. C., Tanenhaus, M. K. \& Kello, C. (1993). Verb-specific constraints in sentence processing: Separating effects of lexical preference from garden-paths. Journal of Experimental Psychology: Learning, Memory and Cognition, 19, 528-553.

von Berger, E., Wulfleck, B., Bates, E. \& Fink, N. (1996). Developmental changes in on-line sentence processing. First Language, 16, 193-222.

Wilson, M. D. (1988). The MRC Psycholinguistic Database: Machine Readable Dictionary, Version 2. Behavioural Research Methods, Instruments and Computers, 20, 6-11.

\section{APPENDIX: TEST SENTENCES}

\section{Action verbs}

1a. The robber/blew open/the safe/with the new lock/and ran off/with the money

$1 \mathrm{~b}$. The robber/blew open/the safe/with dynamite/and ran off/with the money

1c. The robber/blew open/a safe/with a new lock/and ran off/with the money

$1 \mathrm{~d}$. The robber/blew open/a safe/with dynamite/and ran off/with the money

2a. The man/painted/the door/with the window/but didn't/paint the handle

$2 b$. The man/painted/the door/with the new brush/but didn't/paint the handle

2c. The man/painted/a door/with a window/but didn't/paint the handle

$2 \mathrm{~d}$. The man/painted/a door/with a new brush/but didn't/paint the handle

3a. The girl/hit/the boy/with curly hair/but he didn't/know why.

3b. The girl/hit/the boy/with her ruler/but he didn't/know why.

3c. The girl/hit/a boy/with curly hair/but he didn't/know why.

$3 \mathrm{~d}$. The girl/hit/a boy/with her ruler/but he didn't/know why.

4a. The gardener/chopped down/the tree/with dead leaves/because he/didn't like it.

4b. The gardener/chopped down/the tree/with an axe/because he/didn't like it.

4c. The gardener/chopped down/a tree/with dead leaves/because he/didn't like it.

4d. The gardener/chopped down/a tree/with an axe/because he/didn't like it.

5a. The girl/drank/the juice/with some ice/and finished/ every last drop.

5b. The girl/drank/the juice/with a straw/and finished/ every last drop.

$5 c$. The girl/drank/a juice/with some ice/and finished/ every last drop.

$5 \mathrm{~d}$. The girl/drank/a juice/with a straw/and finished/ every last drop.

6a. The monkey/ate/the banana/with the bruise/and everyone/laughed at him.

$6 \mathrm{~b}$. The monkey/ate/the banana/with the fork/and everyone/laughed at him.

$6 c$. The monkey/ate/a banana/with a bruise/and everyone/laughed at him.

$6 \mathrm{~d}$. The monkey/ate/a banana/with a fork/and everyone/laughed at him.

7a. The fireman/smashed down/the door/with the rusty lock/but there was/too much smoke.

7b. The fireman/smashed down/the door/with the heavy axe/but there was/too much smoke.

7c. The fireman/smashed down/a door/with a rusty lock/but there was/too much smoke.

$7 \mathrm{~d}$. The fireman/smashed down/a door/with a heavy axe/but there was/too much smoke. 
8a. The girl/cut/the paper/with nice colours/because she was/wrapping a present.

8b. The girl/cut/the paper/with sharp scissors/because she was/wrapping a present.

$8 \mathrm{c}$. The girl/cut/some paper/with nice colours/because she was/wrapping a present.

$8 \mathrm{~d}$. The girl/cut/some paper/with sharp scissors/because she was/wrapping a present.

9a. The man/patted/the ostrich/with the broken wing/but it didn't like/being touched.

9b. The man/patted/the ostrich/with much excitement/but it didn't like/being touched.

9c. The man/patted/an ostrich/with a broken wing/but it didn't like/being touched.

9d. The man/patted/an ostrich/with much excitement/but it didn't like/being touched.

10a. The boy/read/the book/with the red cover/and enjoyed it/until the end.

10b. The boy/read/the book/with his grandmother/and enjoyed it/until the end.

10c. The boy/read/a book/with a red cover/and enjoyed it/until the end.

$10 \mathrm{~d}$. The boy/read/a book/with his grandmother/and enjoyed it/until the end.

11a. The boy/broke/the toy/with sparkling lights/but it was/ an accident.

$11 \mathrm{~b}$. The boy/broke/the toy/with the hammer/but it was/ an accident.

11c. The boy/broke/a toy/with sparkling lights/but it was/ an accident.

$11 \mathrm{~d}$. The boy/broke/a toy/with a hammer/but it was/ an accident.

12a. The cleaner/wiped/the window/with the smudge/but it was/still very dirty.

12b. The cleaner/wiped/the window/with the cloth/but it was/still very dirty.

12c. The cleaner/wiped/a window/with a smudge/but it was/still very dirty.

$12 \mathrm{~d}$. The cleaner/wiped/a window/with a cloth/but it was/still very dirty.

\section{Psychological predicates and verbs of perception}

13a. The boy/hoped for/the present/with the big red ribbon/and was happy/when he got it.

13b. The boy/hoped for/the present/with anticipation/and was happy/when he got it.

13c. The boy/hoped for/a present/with a big red ribbon/and was happy/when he got it.

13d. The boy/hoped for/a present/with anticipation/and was happy/when he got it.

14a. The boy/listened to/the girl/with the blonde hair/but he/didn't trust her.

14b. The boy/listened to/the girl/with suspicion/but he/didn't trust her.

14c. The boy/listened to/a girl/with long blonde hair/but he/didn't trust her.

$14 \mathrm{~d}$. The boy/listened to/a girl/with suspicion/but he/didn't trust her.

15a. The spy/saw/the cop/with the big machine gun/but the cop/didn't see him.

15b. The spy/saw/the cop/ with the binoculars/but the cop/didn't see him.

15c. The spy/saw/a cop/with a big machine gun/but the cop/didn't see him.

$15 \mathrm{~d}$. The spy/saw/a cop/ with some binoculars/ but the cop/didn't see him.

16a. The man/knew/the code/with eight numbers/but he/didn't use it.

16b. The man/knew/the code/with certainty/but he/didn't use it.

16c. The man/knew/a code/with eight numbers/but he/didn't use it.

$16 \mathrm{~d}$. The man/knew/a code/with certainty/but he/didn't use it.

17a. The girl/heard/the song/with odd words/and she/really liked it.

17b. The girl/heard/the song/with much glee/and she/really liked it.

17c. The girl/heard/a song/with odd words/and she/really liked it.

$17 \mathrm{~d}$. The girl/heard/a song/with much glee/and she/really liked it.

18a. The boy/tasted/the hamburger/with bacon/and quickly/ate it all.

18b. The boy/tasted/the hamburger/with delight/and quickly/ate it all.

18c. The boy/tasted/a hamburger/with bacon/and quickly/ate it all.

$18 \mathrm{~d}$. The boy/tasted/a hamburger/with delight/and quickly/ate it all. 


\title{
VOLUME 27 ISSUE 1
}

19a. The girl/looked at/the boy/with the basketball/because she/wanted to play.

19b. The girl/looked at/the boy/ with much excitement/because she/wanted to play.

19c. The girl/looked at/a boy/with a basketball/because she/wanted to play.

$19 \mathrm{~d}$. The girl/looked at/a boy/ with much excitement/because she/wanted to play.

20a. The lady/loved/the man/with the fast car/but didn't like/his haircut.

20b. The lady/loved/the man/with all her heart/but didn't like/his haircut.

20c. The lady/loved/a man/with a fast car/but didn't like/his haircut.

20d. The lady/loved/a man/with all her heart/but didn't like/his haircut.

21a. The girl/smelled/the cake/with the icing/but she/didn't eat it.

$21 \mathrm{~b}$. The girl/smelled/the cake/with much delight/but she/didn't eat it.

21c. The girl/smelled/a cake/with pink icing/but she/didn't eat it.

21d. The girl/smelled/a cake/with much delight/but she/didn't eat it.

22a. The sailor/looked for/the ship/with the purple sails/but couldn't/see anything.

22b. The sailor/looked for/the ship/with his telescope/but couldn't/see anything.

22c. The sailor/looked for/a ship/with two purple sails/but couldn't/see anything.

$22 \mathrm{~d}$. The sailor/looked for/a ship/with his telescope/but couldn't/see anything.

23a. The scientist/looked at/the insect/with the little wings/and decided to/study it. 23b. The scientist/looked at/the insect/with the microscope/and decided to/study it. 23c. The scientist/looked at/an insect/with two little wings/and decided to/study it. 23d. The scientist/looked at/an insect/with the microscope/and decided to/study it.

24a. The lady/saw/the bus/with the mean bus driver/but it/didn't stop.

24b. The lady/saw/the bus/with anticipation/but it/didn't stop.

24c. The lady/saw/a bus/with a mean bus driver/but it/didn't stop.

24d. The lady/saw/a bus/with anticipation/but it/didn't stop.

\section{ADDRESS FOR CORRESPONDENCE}

\author{
Dr Evan Kidd \\ School of Psychological Sciences, The University of Manchester, \\ Oxford Road, Manchester M13 9PL, UK \\ E: evan.j.kidd@manchester.ac.uk
}

